\title{
线段自映射的非游荡集等于周期 点集的一个充要条件
}

\author{
周 作 领 \\ (暨 南 大 学)
}

1. 本文我们继续讨论线段自映射产生的动力系统问题.

设 $f \in C^{0}(I, I)$, 用 $P(f)$ 和 $Q(f)$ 分别表示 $f$ 的周期点集和非游荡集. 其它有关定义, 名词和符号见另文 ${ }^{1)}$. 我们的目的是证明下述

定理 1 设 $f \in C^{0}(I, I)$. 则 $Q(f)=P(f)$ 当且仅当 $P(f)$ 是闭的.

我们首先证明下述定理 2 和推论,然后给出定理 1 的证明.

定理 2 设 $f \in C^{0}(I, I)$. 若 $f$ 有异状点, 则 $\overline{P(f)} \neq P(f)$.

推论 设 $f \in C^{0}(I, I)$. 若 $\overline{P(f)}=P(f)$, 则 $f$ 无异状点.

2. 上述定理的证明基于下面几个引理, 叙述如下:

引理 1 设 $f \in C^{0}(I, I)$ 和 $p \in I$ 是 $f$ 的不动点. 如果 $W_{+}^{\prime \prime}(p, f) \not \subset W^{\prime \prime}(p, f,+)$, 则 (1) $W_{-}^{u}(p, f) \neq\{p\} ;$ （2）存在 $y \in W_{-}^{u}(p, f)$, 使 $\left.f(y)<y<p ; （ 3\right) f$ 在 $\overline{W_{-}^{u}(p, f)}$ 上 的最大值大于 $p$. 这个引理的证明已在另一文中给出 ${ }^{2)}$.

引理 2 设 $f \in C^{0}(I, I)$ 和 $p \in I$ 是 $f$ 的不动点. 则 $W_{-}^{u}(p, f) \subset W^{u}(p, f,-)$ 或 $W_{-}^{u}(p, f) \subset W^{u}(p, f,+)$.

证 据定义, $W^{u}(p, f,-) \cup W^{u}(p, f,+)=W^{\prime \prime}(p, f)$. 又 $W_{-}^{u}(p, f), W^{u}(p, f,-)$ 和 $W^{u}(p, f,+)$ 都是连通的且都包含 $p$. 若 $W_{-}^{u}(p, f) \not \subset W^{u}(p, f,-)$ 且对任意 $x \in W^{u}(p$, $f)-W^{u}(p, f,-)$, 那么 $x \in W^{u}(p, f,+)$. 所以 $W_{-}^{u}(p, f) \subset W^{u}(p, f,+)$.

引理 3 设 $f \in C^{0}(I, I)$ 和 $p \in I$ 是 $f$ 的不动点. 如果 $W_{+}^{u}(p, f) \not \subset W^{u}(p, f,+)$, 则 存在内点 $q \in W_{-}^{u}(p, f)$, 使 $q \neq p, f(q)=p$ 和 $q \in \overline{P(f)} \subset Q(f)$.

证 据引理 1 , 存在 $y, z \in W_{-}^{u}(p, f)$, 使 $f(y)<p, f(z)>p$. 据引理 2 , 分两种情形证明.

(1) $W^{*}(p, f) \subset W^{\prime \prime}(p, f,-)$.

用 $K_{y}$ 表 $W_{-}^{u}(p, f)$ 的包含 $y$ 的最大连通开子集，满足条件 $f(x)<p, \forall x \in K_{y}$. 易见 $\bar{K}_{y}$ 至少有一个边界点是 $W_{-}^{u}(p, f)$ 的内点, 记 $q_{y}$ 是 $\bar{K}_{y}$ 的同时是 $W_{-}^{u}(p, f)$ 的内点的边界点. 易 于看出 $q_{y} \neq p, f\left(q_{y}\right)=p$, 且对 $q_{y}$ 的满足条件 $\overline{v\left(q_{y}\right)} \subset W_{-}^{u}(p, f)$ 的任意邻域 $v\left(q_{y}\right), f(v$ $\left.\left(q_{y}\right)\right)$ 包含某个 $\nu_{-}(p)=(s, p] \subset I$. 注意,在假设条件下, $0<p<1$.

因 $W_{-}^{u}(p, f) \subset W^{u}(p, f,-)$, 显然存在 $k>0$, 使 $\overline{v\left(q_{y}\right)} \subset f^{k}\left(v_{-}(p)\right) \subset f^{k}\left(f\left(v\left(q_{y}\right)\right)\right)=$ $f^{k+1}\left(v\left(q_{y}\right)\right) \subset f^{k+1}\left(\overline{v\left(q_{y}\right)}\right)$. 因为 $q_{y} \bar{\epsilon} P(f)$, 据文献[1]引理 5 , 在 $\overline{v\left(q_{y}\right)}$ 上存在 $f$ 的异于 $q_{y}$ 的

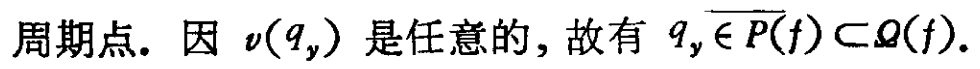

本文 1981 年 1 月 24 日收到.

1) 周作领,数学年刊,将发表.

2) 周作领、刘开金,线段自映射有开状点的一个充要条件,数学进展,将发表. 
(2) $\cdot W^{*}=(p, f) \subset W^{\prime \prime}(p, f,+)$.

与 (1) 相类, 可以证明, 存在 $q_{x} \in W^{*}(p, f)$, 使 $q_{x} \neq p, f\left(q_{x}\right)=p$ 和 $q_{x} \in \overline{P(f)} \subset Q(f)$.

定理 2 的证明: 设 $f$ 有异状点. 我们将证明 $f$ 有异状点 $q$ 满足条件 $q \in \overline{P(f)} \subset Q(f)$. 因 为异状点不是周期的, 故 $q \bar{E} P(f)$. 这样就完成了定理的证明.

正如我们已经证明过的一个定理一样 ${ }^{1)}$, 不失普遍性, 我们可以假设 $f$ 在 $W^{u}(p, f)$ 中有 异状点,其中 $p \in I$ 是 $f$ 的一个不动点.

设 $p \in I$ 是 $f$ 的不动点, 且 $f$ 有异状点 $x \in W_{+}^{u}(p, f), x \neq p, f(x)=p$. 我们证明存在 这样的异状点 $q \in W^{\prime \prime}(p, f), q \neq p, f(q)=p$, 且 $q \in \overline{P(f)} \subset Q(f)$. 分四种情形.

(1) $f\left(W_{+}^{\prime \prime}(p, f)\right)=\{p\}$.

由假设, $W_{+}^{*}(p, f) \neq\{p\}$, 不难看出 $W^{u}(p, f,+)=\{p\}$. 因此 $W_{+}^{*}(p, f) \not \subset W^{u}(p, f$ ， + ). 据引理 3, 存在异状点 $q \in W^{\prime \prime}(p, f), q \neq p, f(q)=p$ 且 $q \in \overline{P(f)} \subset Q(f)$. 这个 $q$ 即 是所求.

(2) $f\left(W_{+}^{*}(p, f)\right) \neq\{p\}$ 和 $f\left(W_{+}^{*}(p, f)\right) \subset W_{+}^{*}(p, f)$.

显然存在 $z \in W_{+}^{u}(p, f)$, 使 $f(z)>p$. 记 $K$ 为 $W_{+}^{u}(p, f)$ 的包含 $z$ 的最大连通开子集, 满足条件 $f(y)>p, \forall y \in K$. 用与引理 3 相同的讨论, 可以假设 $w$ 是 $K$ 的一个同时是 $W_{+}^{*}(p$,

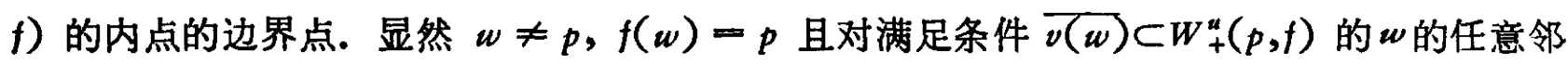
域 $v(w), f(v(w))$ 包含某个 $v_{+}(p)=[p, s) \subset I$.

如果 $W_{+}^{\prime \prime}(p, f) \subset W^{u}(p, f,+)$, 用引理 3 的方法易于证明, $w \in \overline{P(f)} \subset Q(f)$. 令 $q=w$ 即是所求. 若 $W_{+}^{\prime \prime}(p, f) \not \subset W^{\prime \prime}(p, f,+)$, 据引理 3 , 存在异状点 $q \in W_{-}^{\prime \prime}(p, f), q \neq p, f(q)=$ $p$ 且 $q \in \overline{P(f)} \subset \mathcal{Q}(f)$. $q$ 即是所求.

(3) $f\left(W_{+}^{\prime \prime}(p, f)\right) \neq\{p\}$ 和 $f\left(W_{+}^{u}(p, f)\right) \subset W_{-}^{u}(p, f)$.

据 $f$ 的连续性, 对任意 $v_{-}(p)$, 存在 $v_{+}(p)$, 使 $f\left(v_{+}(p)\right) \subset v_{-}(p)$. 依定义, 显然 $W^{u}(p$, $f,+) \subset W^{\prime \prime}(p, f,-)$. 用 (2) 的方法可以证明, 存在 $q \in W_{+}^{u}(p, f), q \neq p, f(q)-p$ 且对满足 条件 $\overline{v(q)} \subset W_{+}^{*}(p, f)$ 的 $q$ 的任意邻域 $v(q), f(v(q))$ 包含某个 $v_{-}(p)$. 类似引理 3 , 容易 看到 $q \in \overline{P(t)} \subset Q(f)$. $q$ 即是所求.

(4) $f\left(W_{+}^{u}(p, f)\right) \cap W_{+}^{u}(p, f) \neq\{p\}$ 和 $f\left(W_{+}^{u}(p, f)\right) \cap W_{-}^{u}(p, f) \neq\{p\}$.

这时显然存在 $y, z \in W_{+}^{*}(p, f)$, 使 $f(y)<p, f(z)>p$. 用引理3的方法易于证明, 存在 异状点 $q \in W_{+}^{u}(p, f), q \neq p, f(q)=p$ 且 $q \in \overline{P(f)} \subset Q(f)$. $q$ 即是所求.

(1)一-(4) 满含假设异状点 $x \in W_{+}^{u}(p, f)$ 的所有可能. 当异状点 $x \in W_{-}^{u}(p, f)$ 时, 证同. 推论显然是定理 2 的直接结果.

定理 1 的证明: 必要部份显然, 因为 $P(f) \subset Q(f)$ 且 $Q(f)$ 是闭的. 充分部份是上述推论 和另文 ${ }^{2}$ 主要定理的直接结果.

致谢: 衰心感谢雾山涛教授对本文写作的热情鼓励和帮助.

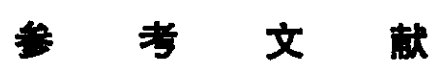

[1] Block, L., Trans. Amer. Math. Soc., 240 (1978), 221-230.

1) 同上页注 2).

2) 周作领,线段自映射的非游荡集等于周期点集的一个充分条件,数学学报,将发表。 